\title{
ON THE REPRESENTATION PROBLEM FOR STATIONARY STOCHASTIC PROCESSES WITH TRIVIAL TAIL FIELD ${ }^{1}$
}

\author{
BY D. L. HANSON \\ Communicated by J. L. Doob, October 30, 1961
}

Let $\left\{X_{n}\right\}$ be a real valued strictly stationary stochastic process on the probability space $(\Omega, \Sigma, P)$ and let $\left\{\xi_{n}\right\}$ be an independent sequence of random variables uniformly distributed on $[0,1]$ where $n=0, \pm 1, \cdots$. When does there exist a function $f$ on the sequence $\left\{\xi_{n}\right\}$ such that the sequences $\left\{X_{n}\right\}$ and $\left\{f\left(T^{n} \xi\right)\right\}$ have the same probability structure where $\xi=\left(\cdots, \xi_{-1}, \xi_{0}, \xi_{1}, \cdots\right)$ and $T \xi$ $=\left(\cdots, \xi_{0}, \xi_{1}, \xi_{2}, \cdots\right)$ (i.e. such that the joint distribution of $X_{i_{1}}, \cdots, X_{i_{k}}$ is the same as the joint distribution of $f\left(T^{i 1} \xi\right), \cdots$, $f\left(T^{i k \xi}\right)$ for all $k$ and all sequences $\left.i_{1}, \cdots, i_{k}\right)$ ?

Let $\Sigma_{n}$ be the smallest $\sigma$-field of subsets of $\Omega$ with respect to which $X_{k}$ is measurable for all $k \leqq n$ and let $\Sigma_{-\infty}=\cap \Sigma_{n} . \Sigma_{-\infty}$ is called the tail field of the process $\left\{X_{n}\right\}$ and is said to be trivial if $A \in \Sigma_{-\infty}$ implies $P(A)=0$ or 1 . It has been shown (see [1] and [2]) that if $\left\{X_{n}\right\}$ is a stationary Markov chain with a denumerable state space and whose tail field is trivial then a representation of the above type holds and in fact $f\left(T^{n} \xi\right)=f\left(\cdots, \xi_{n-1}, \xi_{n}\right){ }^{2}$

By use of a fairly simple transformation an arbitrary stationary process $\left\{X_{n}\right\}$ with trivial tail field can be converted to a stationary Markov process $\left\{Y_{n}\right\}$ with trivial tail field and from which the $\left\{X_{n}\right\}$ process can be recovered. Thus the seeming preoccupation with Markov processes.

The following theorem generalizes Rosenblatt's results to a class of Markov process with nondenumerable state space. $\bar{P}$ is the stationary measure induced by the process on the state space and $P_{X}\left(A^{\prime}\right)$ is the stationary conditional probability that $X_{n} \in A^{\prime}$ given $X_{n-1}=X$.

THEOREM. Let $\left\{X_{n}\right\}, n=0, \pm 1, \cdots$ be a real stationary Markov process such that

(i) $\Sigma_{-\infty}$ is trivial.

(ii) There exist Borel subsets $A$ and $B$ of the state space and a nonnegative measure $\phi$ on the state space such that $\bar{P}(B)>0, \phi(A)>0$, and for all $X \in B$ and $A^{\prime} \subset A$ we have $P_{X}\left(A^{\prime}\right) \geqq \phi\left(A^{\prime}\right)$.

\footnotetext{
1 This work was performed under the auspices of the United States Atomic Energy Commission.

${ }^{2}$ A stationary Markov chain with denumerable state space has a trivial tail field if and only if it is ergodic and aperiodic.
} 
Then if $\left\{\xi_{n}\right\}$ is an independent sequence of random variables uniformly distributed $[0,1]$ there exists a function $g=g\left(\cdots, \xi_{-1}, \xi_{0}\right)$ such that the sequences $\left\{X_{n}\right\}$ and $\left\{g\left(\cdots, \xi_{n-1}, \xi_{n}\right)\right\}$ have the same probability structure.

COROLlary 1. In the above theorem it is sufficient to replace condition (ii) with

(iia) The state space of $\left\{X_{n}\right\}$ has an atom under the stationary probability $\bar{P}$.

Corollary 2. If $\left\{X_{n}\right\}$ is a stationary ergodic aperiodic Markov chain with a denumerable state space then conditions (i) and (ii) hold and the above theorem is true.

Detailed proofs will appear elsewhere.

\section{REFERENCES}

1. M. Rosenblatt, Stationary processes as shifts of functions of independent random variables, J. Math. Mech. 8 (1959), 665-681.

2. - Stationary Markov chains and independent random variables, J. Math. Mech. 9 (1960), 945-949.

\section{Sandia CoRporation}

